\title{
Shear Rigidity Percolation in 2D Solid-Liquid Composites
}

\author{
Minyao Zhou and Ping Sheng \\ Exxon Research and Engineering Company, Route 22 East, Annandale, New Jersey 08801
}

(Received 26 July 1993)

\begin{abstract}
Shear rigidity percolation is studied on a simple square lattice where a fraction $p$ of the area is filled by an elastic solid, and the rest by liquid. The presence of the liquid is shown to yield a new shear critical exponent $\tau$ at the percolation threshold. Through finite-size scaling calculations the value of $\tau$ is found to be $1.2 \pm 0.1$, while similar calculations on solid-vacuum composites yield the $2 \mathrm{D}$ elastic critical exponent $T=3.4 \pm 0.2$, in excellent agreement with prior results. The underlying physics of the solidliquid rigidity percolation is discussed.
\end{abstract}

PACS numbers: $62.20 .-\mathrm{x}, 46.30 . \mathrm{Cn}, 61.43 .-\mathrm{j}$

It is now well established that the elastic and the electrical properties of a composite have different critical behaviors near the percolation threshold. This difference has been especially well-documented for the 2D percolation problem, where both experiment [1-4] and theory [5-10] show that the elastic moduli of a solid-vacuum composite vary as $\left(p-p_{c}\right)^{T}$ near the percolation threshold $p_{c}$, where $p$ is the solid fraction and $T \simeq 3.4-3.6$ for percolation on a lattice and $T \simeq 4.95$ for the continuum percolation case where the bond strengths can have a singular distribution [4]. This is in sharp contrast to the electrical percolation behavior [11], where the conductivity of a $2 \mathrm{D}$ metal-insulator composite is known to vary as $\left(p-p_{c}\right)^{t}$ near $p_{c}$, with $t=1.1-1.3$.

A distinct feature of the elastic percolation behavior is that both the effective bulk modulus $\bar{\kappa}$ and the shear modulus $\bar{\mu}$ have the same critical exponent. The physics of this identity was elucidated by Kantor [6], who showed that by considering the elasticity of the percolation cluster backbone as that of a tortuous elastic chain, one can easily deduce that the compression response and the shear response must scale similarly as a function of the chain length, thus ensuring the equality of the critical exponents for $\bar{\kappa}$ and $\bar{\mu}$. However, since compression and shear represent 2 independent degrees of freedom for the elastic response of a material, an obvious alternate elastic percolation problem is that of the solid-liquid composites, in which only $\bar{\mu}$ vanishes at $p_{c}$. We show in this work that although liquid has no shear modulus, its presence can nevertheless induce an entirely new shear rigidity percolation behavior. Below we first discuss the physics of the problem. Numerical finite-size scaling results are then presented for both the solid-liquid and solid-vacuum composites. While the solid-vacuum calculations recover the value of $T=3.4 \pm 0.2$ and the ratio $\bar{C}_{11} / \bar{\mu}=3.4 \pm 0.2$ at the percolation threshold, where in $2 \mathrm{D} \bar{C}_{11}=\bar{\kappa}+\bar{\mu}$, exactly similar calculations with vacuum replaced by liquid give $\bar{\mu} \propto\left(p-p_{c}\right)^{\tau}$, with $\tau=1.2 \pm 0.1$. A new feature of our calculations is the consideration of continuum elastic solid and liquid instead of discrete springs with bondbending restoring force as our percolating components. This has the advantage that there is no ambiguity about the value of the elastic percolation threshold, since the geometric, electric (if solid is assumed to be conducting and the liquid and vacuum to be insulating), and elastic percolation thresholds all coincide and occur at $p_{c}$ $=0.5927$.

Consider a 2D checkerboard in which each square can be either solid, with probability $p$, or liquid (or empty), with probability $1-p$. Because of the underlying square symmetry of the system, there are three effective elastic constants. However, in this work we will be concerned only with two, $\bar{C}_{11}$ and $\bar{\mu}$. At $p>p_{c}$, liquid cannot form a continuous infinite network. Therefore, it is inevitably segmented into isolated pockets. As a liquid pocket can offer resistance to shear deformation only when its area is altered, one can envision that in the case of the percolation cluster geometry, the application of a macroscopic shear strain $\epsilon$ to the sample would result in area changes of the pockets that are first order in $\epsilon$, since the random geometry would make it very unlikely to have it otherwise. That means the liquid can exert a force on the connected solid network through the perimeters of the pockets, and from the point of view of the solid network this would introduce a nonlocal means of stress transmission and a net stiffening of the composite against shear deformation. As $p$ approaches $p_{c}$ from above, the average pocket size in the percolation cluster is known to grow in a power-law manner [11]. Since the pocket size controls the range of stress transmission through the liquid, it is thus expected that the presence of the liquid would introduce a new critical behavior to the shear rigidity percolation problem.

To determine the precise value of the new shear rigidity percolation exponent, we use the well-established finite-size scaling method $[8,12,13]$ in which the effective moduli of a strip of width $L$ (in units of the square size) and length $N \gg L$ are exactly solved numerically through the finite difference approach. Here the width of the strip is defined to be in the $y$ direction, and the length in the $x$ direction. The value of $p$ is fixed at $p_{c}=0.5927$. As the width $L$ of the strip increases, the effective moduli are expected to vary as $L^{-(T / v)}$ for the solid-vacuum composites and as $L^{-(\tau / v)}$ for the shear modulus of the solid- 
liquid composites, where $v=\frac{4}{3}$ is the correlation length exponent and $L$ is assumed to be sufficiently large.

The starting point of our calculation is the $2 \mathrm{D}$ stressstrain relations:

$$
\begin{aligned}
\tau_{x x} & =(\kappa+\mu) \frac{\partial u_{x}}{\partial x}+(\kappa-\mu) \frac{\partial u_{y}}{\partial y}, \\
\tau_{y y} & =(\kappa+\mu) \frac{\partial u_{y}}{\partial y}+(\kappa-\mu) \frac{\partial u_{x}}{\partial x} \\
\tau_{x y} & =\mu\left(\frac{\partial u_{x}}{\partial y}+\frac{\partial u_{y}}{\partial x}\right) .
\end{aligned}
$$

Here $\tau_{x x}, \tau_{y y}, \tau_{x y r}\left(=\tau_{y x}\right)$ are the three components of the stress tensor, and $u_{x}, u_{y}$ are the displacements in the two directions. The shear modulus $\mu$ is set equal to zero for the liquid. For both liquid and solid, $\kappa-\mu$ is taken to be 1 for simplicity. Given the stress tensor, the force balance equation is simply

$$
\begin{aligned}
& \frac{\partial \tau_{x x}}{\partial x}+\frac{\partial \tau_{x y}}{\partial y}=0, \\
& \frac{\partial \tau_{y y}}{\partial y}+\frac{\partial \tau_{x y}}{\partial x}=0 .
\end{aligned}
$$

At the liquid-solid interfaces, the boundary conditions are the continuity of normal stress, $\tau_{x y}=0$, and the continuity of normal displacement. The boundary condition at the solid-vacuum interface is simply the vanishing of all the stress components.

For the purpose of finite-difference numerical calculations, the discretization of each square is done with $\Delta x=\Delta y=\frac{1}{4}$ (sixteen discretization units per square) and a staggered-grid scheme [14], where $\tau_{x x}$ and $\tau_{y y}$ are defined at the center for each discretization unit, $\tau_{x y}$ is defined at the corners, and the displacements are defined at the midpoints of the sides of every $\frac{1}{4} \times \frac{1}{4}$ square. To evaluate the compressional modulus $\bar{C}_{11}$ or the shear modulus $\bar{\mu}$ of the composite strip, a uniform $u_{y}=0.1$ or $u_{x}=0.1$ is imposed on the top edge of the strip, while the bottom edge is held fixed. The value of $L$ is varied from 4 to 40 , while $N$ varies from 100 to 2000 . When $N$ is large, the number of configurational average can be reduced. This is because the cluster is not continuous in the lengthwise direction, so a large $N$ can contain many separate (and parallel) clusters. In that case a single configuration is equivalent to averaging over many smaller- $N$ configurations. Once the problem is solved numerically, the modulus is defined by the total elastic energy $E$ as follows. Since $E=$ (the net force $F$ on the top edge of the strip $) \times($ applied displacement $) / 2$, and $F=($ effective modulus $\bar{C}_{11}$ or $\left.\bar{\mu}\right) \times($ applied displacement $) / L$, it follows that effective modulus $=2 E L /(\text { applied displacement })^{2}$. The total elastic energy is obtained from the numerical solution as one-half the sum of the product of the stress with strain over all the discretized units. The main results of the calculations are shown in Fig. 1 , where $\log \bar{\mu}$ is plotted as a function of $\log L$. The value of $\mu$ for the solid is tak-

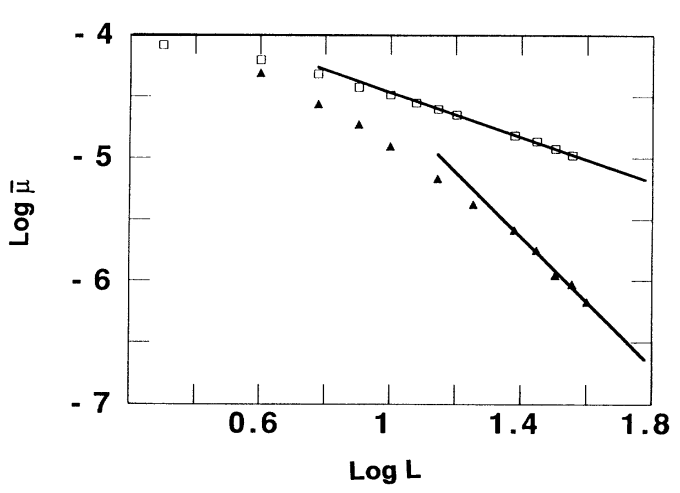

FIG. 1. Variation of the effective shear modulus $\bar{\mu}$ with $L$, plotted on the log-log scale. Solid triangles are for the solidvacuum composites and open squares are for the solid-liquid composites. $\mu$ for the solid is taken to be 0.5 (in units where $\kappa-\mu=1)$. The asymptotic slopes of the least-squares fitted lines are $-2.6 \pm 0.3$ and $-0.91 \pm 0.02$, for which one obtains $T=3.4 \pm 0.2$ and $\tau \simeq 1.2 \pm 0.1$.

en to be 0.5 . It is seen that for the solid-vacuum composites the asymptotic regime is reached at $L \geq 24$. The slope obtained from the region of $L=24-40$ gives $T=3.4 \pm 0.2$, in excellent agreement with all previous theoretical lattice calculations as well as experimental measurements. For the solid-liquid composites, on the other hand, the asymptotic slope is much less than the solid-vacuum case. The slope in that case yields $\tau=1.2 \pm 0.1$. This is noted to coincide very closely with the electrical percolation exponent. However, the underlying physics is very different in the two cases. It is seen that the stiffening effect due to the liquid pockets is dominant over the solid backbone by itself. In this regard it should also be noted that in our model there is no stressinduced scalar elastic energy term as proposed by Alex-

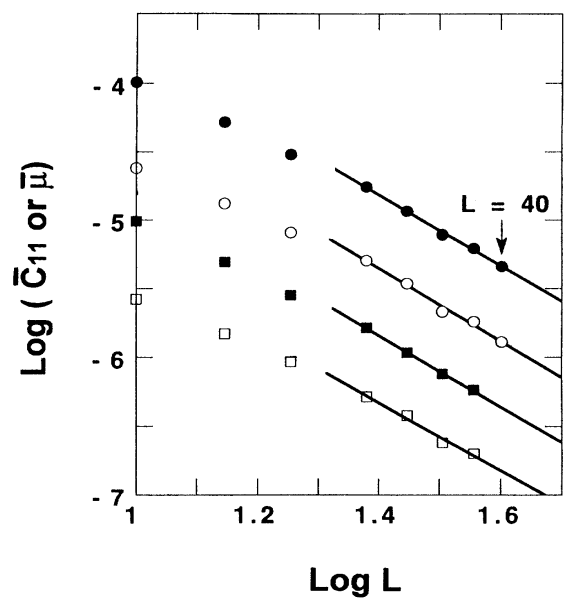

FIG. 2. Variation of $\bar{C}_{11}$ and $\bar{\mu}$ as a function of $L$, plotted on the log-log scale. Two sets of data are shown, one for $\mu=1.0$ (circles) and one for $\mu=0.1$ (squares). The solid symbols are for $\bar{C}_{11}$; the open symbols are for $\bar{\mu}$. 


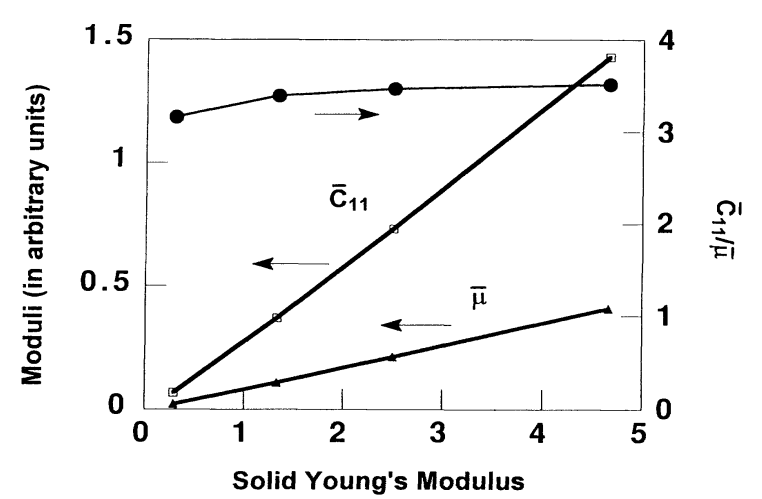

FIG. 3. Variation of $\bar{C}_{11}$ and $\bar{\mu}$ with the Young's modulus of the solid material for the solid-vacuum composite at $p=p_{c}$ and $L=40$. Excellent linear relations are seen. The ratio $\bar{C}_{11} / \bar{\mu}$ is plotted on top (right scale). It is seen that the ratio is almost invariant with respect to the Young's modulus and has the average value of $3.4 \pm 0.2$.

ander [15]. However, the final critical exponent is nevertheless very electrical-like.

For the solid-vacuum composites, the equality of the $\bar{C}_{11}$ exponent and the $\bar{\mu}$ exponent is demonstrated by the results shown in Fig. 2. Two sets of data are plotted, one for $\mu=1.0$ and the other one for $\mu=0.1$. All four data sets show almost identical asymptotic slopes. Leastsquares fits of the data points from $L=24-40$ yield $T=3.46 \pm 0.08 \quad(\mu=1$ case $)$ and $3.44 \pm 0.06 \quad(\mu=0.1$ case) for $\bar{C}_{11}$, and $T=3.52 \pm 0.15(\mu=1$ case $)$ and 3.3 $\pm 0.23(\mu=0.1$ case $)$ for $\bar{\mu}$.

The equality of the exponents means that there is a constant $\bar{C}_{11} / \bar{\mu}$ ratio at the percolation threshold of solid-vacuum composites. Bergman has performed a simulation on a hexagonal lattice and obtained the ratio $\bar{C}_{11} / \bar{\mu}=3.5$ [8]. By arguing that the $\bar{C}_{11} / \bar{\mu}$ is directly linked to the ratio of the anisotropic radii of gyration for singly connected bonds in a percolation cluster, Kantor has obtained a very similar value [6], $3.4 \pm 0.2$ for $\bar{C}_{11} / \bar{\mu}$. However, renormalization calculations on a Sierpinski gasket has yielded $\bar{C}_{11} / \bar{\mu}=3[16]$. As a side calculation of the solid-vacuum composites, we have carried out an evaluation of the critical $\bar{C}_{11} / \bar{\mu}$ ratio as a function of the solid material parameters in the continuum elastic case. In order for the ratio to be independent of the material parameters, the two effective modulus must vary in the same way as a function of the solid material parameters. In Fig. 3 it is shown that, indeed, $\bar{C}_{11}$ and $\bar{\mu}$ are both linear functions of the solid Young's modulus. Physically, this variation can be justified by Kantor's argument that in a random network, bending displacement rather than compression is the dominant local distortion. If that is the case, and we regard the links as $1 \mathrm{D}$-like, then the only parameter relating to the bending force displacement is $Y /\left(1-\sigma^{3}\right)$, where $Y$ is the Young's modulus and $\sigma$ the Poisson ratio. The ratio of the slopes in Fig. 3 gives $\bar{C}_{11} / \bar{\mu}=3.4 \pm 0.2$, in excellent agreement with prior nu- merical results. While $\bar{C}_{11} / \bar{\mu}$ is demonstrated to be a constant, it has been argued that the value of the constant could be dependent on the lattice type [17]. This has been demonstrated on both triangular and square lattices with nearest- and next-nearest-neighbor central forces [18]. It is noted that the conclusion here differs from Kantor's claim of universality for the $\bar{C}_{11} / \bar{\mu}$ ratio. However, the models used in demonstrating the two points of view are also different. For the solid-liquid composites, this ratio is always $\infty$ because $\bar{C}_{11}$ does not vanish at the threshold.

In summary, we have shown through both physical arguments and numerical finite-size scaling calculations that the 2D solid-liquid composites have a new shear critical exponent $\tau$ that is much smaller in value than the elastic critical exponent $T$. Based on our physical understanding of the role of the liquid, the same effect can occur in 3D composites if there is a considerable amount of isolated liquid-filled pores. However, the liquid effect would be minimal if the liquid forms a separate infinite network so that flow is possible. A particularly intriguing case is one in which both trapped liquid pockets and liquid networks coexist. Recent experiment on the shear rigidity percolation of emulsions [19] presents exactly such a scenario. It is speculated that the effect of the trapped liquid may still be important in that case, and the shear critical behavior could therefore depart significantly from that expected from 3D elastic percolation.

[1] C. Allain, J. C. Charmet, M. Clement, and L. Limat, Phys. Rev. B 32, 7552 (1985).

[2] L. C. Allen, B. Golding, and W. H. Haemmerle, Phys. Rev. B 37, 3710 (1988).

[3] D. Deptuck, J. P. Harrison, and P. Zawadzki, Phys. Rev. Lett. 54, 913 (1985).

[4] C. J. Lobb and M. G. Forrester, Phys. Rev. B 35, 1899 (1987).

[5] Y. Kantor and I. Webman, Phys. Rev. Lett. 52, 1891 (1984).

[6] Y. Kantor, J. Phys. A 17, L843 (1984).

[7] S. Feng and M. Sahimi, Phys. Rev. B 31, 1671 (1985).

[8] D. J. Bergman, Phys. Rev. B 31, 1696 (1985).

[9] S. Feng, P. N. Sen, B. I. Halperin, and C. Lobb, Phys. Rev. B 30, 5386 (1984).

[10] L. Benguigui, Phys. Rev. Lett. 53, 2028 (1984).

[11] D. Stauffer, Introduction to Percolation Theory (Taylor \& Francis, London, 1985).

[12] C. J. Lobb and D. J. Frank, J. Phys. C 12, L827 (1979).

[13] B. Derrida and J. Vannimenus, J. Phys. A 15, L557 (1982).

[14] B. Fornberg, SIAM J. Numer. Anal. 27, 904 (1990).

[15] S. Alexander, J. Phys. (Paris) 45, 1939 (1984).

[16] D. J. Bergman and Y. Kantor, Phys. Rev. Lett. 53, 511 (1984).

[17] E. L. Garboczi and M. F. Thorpe, Phys. Rev. B 31, 7276 (1985).

[18] E. L. Garboczi and M. F. Thorpe, Phys. Rev. B 33, 3289 (1986).

[19] D. Weitz (private communication). 